\title{
Empiema pleural en 343 casos con tratamiento quirúrgico: características, resultados inmediatos y factores asociados a morbilidad y mortalidad*
}

\author{
Drs. ROBERTO GONZÁLEZ L. ${ }^{1,2}$, RAFAEL PRATS M. ${ }^{1,2}$, DAVID LAZO P., ${ }^{1,2}$, ANDRÉS JADUE T. ${ }^{2}$, \\ GERARDO MORDOJOVICH R. ${ }^{1,2}$, RAIMUNDO SANTOLAYA C. ${ }^{1,2}$, PATRICIO RODRÍGUEZ D. ${ }^{1,2}$

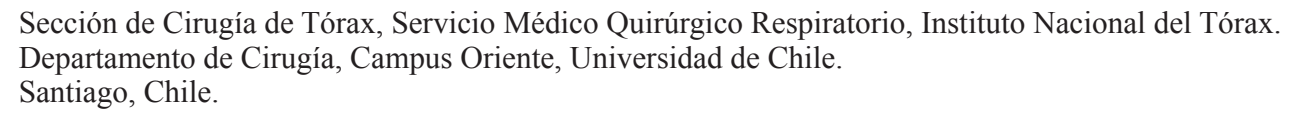

\begin{abstract}
\section{Pleural empyema. Retrospective review of 343 patients}

Background: Pleural empyema still has a dismal prognosis. Aim: To describe features and prognostic factors of patients with pleural empyema subjected to surgical procedures. Material and Methods: Retrospective review of 343 patients with pleural empyema (mean age 52 years, $71 \%$ males), that were subjected to any type of surgical procedure. Criteria for diagnosis of empyema were a positive culture, a fluid $\mathrm{pH}$ of less than 7.2 or a compatible macroscopic appearance of the fluid. Results: Empyema was secondary to pneumonia in 243 patients $(71 \%)$, secondary to surgical procedures in 41 patients $(12 \%)$, secondary to trauma in 19 patients $(5.5 \%)$, secondary to a lung abscess in 10 patients $(3 \%)$, tuberculous in 10 patients $(3 \%)$, neoplastic in two cases $(0.6 \%)$, secondary to pneumothorax in 2 cases $(0.6 \%)$ and of unknown origin in 13 patients (4\%). A microorganism was isolated from pleural fluid in 89 patients $(26 \%)$. The surgical procedures performed were 251 decortications by thoracotomy $(73 \%), 70$ pleurotomies $(20 \%), 11$ video assisted surgeries $(3 \%)$, seven decortications with lung resections and four fenestrations. Complications were recorded in 104 patients (30\%), 29 patients were re-operated (8.5\%) and 31 died (10\%), all due to sepsis. Conclusions: The main cause of pleural empyema is pneumonia. In most patients, microorganisms are not isolated from pleural fluid, thoracotomy with decortication is the most frequent surgical procedure. There is a high rate of complications and mortality.
\end{abstract}

Key words: Empyema, thoracotomy, pneumonia.

\section{Resumen}

Objetivos: Describir características, resultados inmediatos y evaluar factores asociados a morbimortalidad de Empiema Pleural (EP) con tratamiento quirúrgico. Método: Revisión retrospectiva. Período: enero 2000 - agosto 2006. Se describen características, resultados inmediatos y factores asociados a morbimortalidad. Se utilizó programa SPSS 15.0. Se consideró significativo $\mathrm{p}<0,05$. Resultados: 343 pacientes,

*Recibido el 30 de julio de 2011 y aceptado para publicación el 16 de agosto de 2011.

No existen conflictos de interés ni apoyo financiero

Correspondencia: Dr. Roberto González L.

José Manuel Infante 717, Santiago, Chile. Fax: 56-2-5754997

rgonzalezlagos@udec.cl 
243 (70,8 \%) hombres (relación 2,4:1), edad promedio 51,7 años. Etiología: 242 (70,6\%) paraneumónico, $41(12,0 \%)$ postquirúrgico, $19(5,5 \%)$ postraumático, 10 (2,9\%) absceso pulmonar, 10 (2,9\%) tuberculoso, $6(1,7 \%)$ neoplásico, $2(0,6 \%)$ neumotórax y $13(3,8 \%)$ desconocido. Se aisló germen en líquido pleural en $89(25,9 \%)$. Se realizaron: $251(73,2 \%)$ decorticaciones por toracotomía, $70(20,4 \%)$ pleurotomías, 11 $(3,2 \%)$ cirugías video-asistidas, $7(2,1 \%)$ decorticaciones con resección pulmonar y $4(1,2 \%)$ fenestraciones. Presentaron complicaciones $104(30,3 \%)$ pacientes. Se reoperaron 29 (8,5\%). Fallecieron 31 (9,6\%), todos por sepsis. Se encontraron variables asociadas a morbi-mortalidad. Conclusiones: El EP tiene como causa más frecuente el empiema paraneumónico seguido de los postoperatorios, en la mayoría no se identifican gérmenes en líquido pleural. La toracotomía con decorticación es el procedimiento quirúrgico más frecuente. El EP tiene una considerable morbi-mortalidad. Se identifican variables asociadas a morbilidad y mortalidad.

Palabras clave: Empiema pleural, patología pleural, cirugía torácica, morbilidad, mortalidad.

\section{Introducción}

A pesar de los constantes avances en las técnicas quirúrgicas, terapias antibióticas, cuidados y soporte postoperatorio, el empiema pleural (EP) mantiene niveles de morbilidad y mortalidad que pueden llegar a ser particularmente elevados en grupos de riesgo ${ }^{1-3}$.

La etiología más frecuente del EP es el empiema paraneumónico, corresponde aproximadamente al 60 a $70 \%$ de todos los casos de $\mathrm{EP}^{4,5}$. Entre otras causas menos frecuentes están los EP postquirúrgicos, tuberculosos, postraumáticos y neoplásicos ${ }^{1-3,6}$.

El EP es más frecuente en edades extremas, pero puede afectar a cualquier grupo etario y se han descrito variables asociadas al desarrollo de éste y a morbi-mortalidad ${ }^{1-5,7}$. La morbilidad de pacientes con EP tratados quirúrgicamente alcanza al 20 a $30 \%$ y la mortalidad comunicada está entre el 3,5\% y el $20 \%{ }^{1-9}$.

En el Reino Unido y en Estados Unidos afecta a más de 65.000 pacientes cada año, con un costo hospitalario anual estimado en 500 millones de dólares $^{1,4,5}$. En la segunda mitad del siglo pasado se produjo una constante y significativa disminución de la mortalidad por EP, sin embargo, en la última década se ha comunicado un aumento en el número de casos y un aumento de la morbi-mortalidad, especialmente de los empiemas paraneumónicos en pacientes con algún grado de inmunosupresión ${ }^{1,4,5,10,11}$.

Los objetivos de nuestra comunicación son describir características, resultados inmediatos y evaluar factores asociados a morbi-mortalidad de EP tratados quirúrgicamente.

\section{Material y Método}

Se realizó una revisión retrospectiva de bases de datos de empiema, de protocolos operatorios y registro de pabellón del Instituto Nacional del Tórax.

El período comprendió desde enero de 2000 hasta agosto de 2006.
Se incluyeron todos los pacientes con diagnóstico de EP y en quienes se realizó algún procedimiento quirúrgico.

Se consideró como EP los casos que cumplían con una o más de las siguientes características de la ocupación pleural:

- Cultivo microbiológico o tinción de Gram positivos.

- $\mathrm{pH}<7,2$.

- Hallazgo macroscópico compatible con empiema.

Se describen: género, edad, procedencia (lugar donde se diagnosticó y trató el EP antes de ser derivado para el procedimiento quirúrgico en nuestro hospital), comorbilidad, etiología, cultivos microbiológicos, gérmenes aislados, procedimientos quirúrgicos (se consideró el procedimiento final o definitivo) y morbi-mortalidad. Para evaluar factores asociados a morbi-mortalidad se seleccionaron 23 variables, se realizó análisis univariado y multivariado cuya variable dependiente fue morbilidad y mortalidad por separado. Se utilizó programa SPSS 15.0. Se consideró significativo $\mathrm{p}<0,05$.

\section{Resultados}

Durante el período estudiado, en el Instituto Nacional del Tórax se realizaron procedimientos quirúrgicos en 343 pacientes con empiema pleural.

La mayoría de los pacientes fueron de género masculino (relación de $2,4: 1$ ) y el promedio de edad fue de $51,7 \pm 17,5$, con un rango entre 15 y 86 años (Tabla 1).

En relación a la procedencia de los pacientes: 20 $(5,9 \%)$ fueron del Instituto Nacional del Tórax y $323(94,1 \%)$ fueron derivados de otros hospitales. De los derivados de otros hospitales, $256(74,6 \%)$ provenían de la Región Metropolitana y 67 (19,5\%) de hospitales de otras regiones de Chile.

Las comorbilidades más frecuentes fueron tabaquismo, hipertensión arterial y diabetes mellitus (Tabla 2). En 37 (10,8\%) pacientes el EP fue en un "ambiente neoplásico", es decir, en pacientes con al- 
Tabla 1. Distribución según género y edad de pacientes con EP tratados quirúrgicamente

\begin{tabular}{|ll|}
\hline Total EP & $\mathbf{3 4 3}$ \\
Género & \\
Masculino & $243(70,8 \%)$ \\
Femenino & $100(29,2 \%)$ \\
Relación M / F & $2,4 / 1$ \\
Edad (años) & \\
Promedio & $51,7 \pm 17,5$ \\
Mediana & 53,0 \\
Rango & $15-86$ \\
\hline
\end{tabular}

Tabla 3. Etiología de EP tratados quirúrgicamente

\begin{tabular}{|c|c|}
\hline Total EP & 343 \\
\hline \multicolumn{2}{|l|}{ Etiología } \\
\hline Paraneumónico & $242(70,6 \%)$ \\
\hline Postquirúrgico* & $41(12,0 \%)$ \\
\hline Postraumático & $19(5,5 \%)$ \\
\hline Absceso pulmonar & $10 \quad(2,9 \%)$ \\
\hline Tuberculosis & $10 \quad(2,9 \%)$ \\
\hline Neoplásico & $6(1,7 \%)$ \\
\hline Neumotórax & $2(0,6 \%)$ \\
\hline Desconocido & $13(3,8 \%)$ \\
\hline
\end{tabular}

*8 casos postquirúrgicos corresponden a pacientes operados primariamente de cirugía torácica en nuestro hospital.

guna neoplasia maligna, ya sea pleuropulmonar o de otro tipo. Además, se describen factores asociados a gravedad al momento del procedimiento quirúrgico, como deterioro de conciencia y deterioro hemodinámico (requerimiento de drogas vaso activas).

Las etiologías más frecuentes de los EP fueron: $242(70,6 \%)$ paraneumónico y $41(12,0 \%)$ postquirúrgico (Tabla 3 ). De los 41 casos de EP postquirúrgico, 8 casos fueron pacientes operados primariamente en nuestro hospital, la mayoría corresponde a EP postquirúrgico derivados de otros hospitales.

Se aisló algún germen en cualquier tipo de cultivo (hemocultivo, expectoración o líquido pleural) en $192(56,0 \%)$ pacientes y en líquido pleural en 89 $(25,9 \%)$, de estos $31(9,0 \%)$ fueron polimicriobianos (Tabla 4). Los gérmenes más frecuentemente aislados fueron el Staphylococcus aureus, Streptococcus spp y Pseudomonas aeruginosa (Tabla 5).

Se realizaron: $251(73,2 \%)$ decorticaciones por toracotomía, $70(20,4 \%)$ pleurotomías, $11(3,2 \%)$ cirugías video-asistidas, 7 (2,1\%) decorticaciones con resección pulmonar y $4(1,2 \%)$ fenestraciones (Tabla 6).

Presentaron complicaciones 104 (30,3\%) pacientes, se reoperaron $29(8,5 \%)$ y $35(10,2 \%)$ requirie-
Tabla 2. Comorbilidades y factores asociados a gravedad en pacientes con EP tratados quirúrgicamente

\begin{tabular}{|lr|}
\hline Comorbilidad & \\
Tabaquismo & $132(38,5 \%)$ \\
Hipertensión Arterial & $92(26,8 \%)$ \\
Diabetes mellitus & $60(17,5 \%)$ \\
Ambiente neoplásico & $37(10,8 \%)$ \\
Factores asociados a gravedad & \\
Deterioro de conciencia & $42(12,2 \%)$ \\
Deterioro hemodinámico & $32(9,3 \%)$ \\
\hline
\end{tabular}

Tabla 4. Estudios microbiológicos en pacientes con EP tratados quirúrgicamente

\begin{tabular}{|lr|}
\hline Total EP & $\mathbf{3 4 3}$ \\
Germen aislado* & \\
Positivo & $192(56,0 \%)$ \\
Negativo & $151(44,0 \%)$ \\
Cultivo de líquido pleural & \\
Negativo & $254(74,1 \%)$ \\
Positivo & $89(25,9 \%)$ \\
Cultivo polimicrobiano & $31(9,0 \%)$ \\
\hline
\end{tabular}

*Germen aislado en cualquier tipo de cultivo: hemocultivo, expectoración o líquido pleural.

Tabla 5. Gérmenes aislados en estudios microbiológicos de líquido pleural en pacientes con EP tratados quirúrgicamente

\begin{tabular}{|ll|}
\hline Germen aislado (en líquido pleural) & \\
\hline Staphylococcus aureus & $77(22,4 \%)$ \\
\hline Streptococcus spp & $39(11,4 \%)$ \\
\hline Pseudomonas aeruginosa & $23(6,7 \%)$ \\
\hline Escherichia coli & $14(4,1 \%)$ \\
\hline Klebsiella pneumoniae & $13(3,8 \%)$ \\
Acinectobacter baumannii & $10(2,9 \%)$ \\
\hline Otros & $18(5,2 \%)$ \\
\hline
\end{tabular}

ron unidad de cuidados intensivos (UCI) en el post operatorio (Tablas 6,7 y 8 ).

Fallecieron $31(9,0 \%)$ pacientes, todos por sepsis.

De las variables seleccionadas para la morbilidad presentaron significación estadística en el análisis multivariado: deterioro hemodinámico; deterioro de conciencia; identificación de germen; cultivo positivo para Staphylococcus aureus, Klebsiella 
Taba 6. Procedimientos quirúrgicos, morbilidad y mortalidad según tipo de procedimiento y total en pacientes con EP tratados quirúrgicamente

\begin{tabular}{|c|c|c|c|}
\hline $\begin{array}{l}\text { Procedimiento } \\
\text { quirúrgico }\end{array}$ & Total (\%) & $\begin{array}{c}\text { Morbilidad } \\
(\%)\end{array}$ & $\begin{array}{l}\text { Mortalidad } \\
\text { (\%) }\end{array}$ \\
\hline $\begin{array}{l}\text { Decorticación por } \\
\text { toracotomía }\end{array}$ & $251 \quad(73,2)$ & $85(33,9)$ & $19(7,6)$ \\
\hline Pleurotomía & $70 \quad(20,4)$ & $11(15,7)$ & $11(15,7)$ \\
\hline $\begin{array}{l}\text { Videotoracoscopia o } \\
\text { video-asistida }\end{array}$ & $11 \quad(3,2)$ & $1 \quad(9,1)$ & 0 \\
\hline $\begin{array}{l}\text { Decorticación más } \\
\text { resección pulmonar }\end{array}$ & $7 \quad(2,1)$ & $5(71,4)$ & $1(14,3)$ \\
\hline Fenestración & $4 \quad(2,1)$ & $2(50,0)$ & 0 \\
\hline Total & $343(100)$ & $104(30,3)$ & $31 \quad(9,0)$ \\
\hline
\end{tabular}

Taba 7. Etiología, morbilidad y mortalidad en pacientes con EP tratados quirúrgicamente

\begin{tabular}{|c|c|c|c|}
\hline & Total & $\begin{array}{c}\text { Morbilidad } \\
(\%)\end{array}$ & $\begin{array}{c}\text { Mortalidad } \\
(\%)\end{array}$ \\
\hline Etiología & 343 & $104(30,3)$ & $31 \quad(9,0)$ \\
\hline Paraneumónico & 242 & $65(26,9)$ & $16(6,6)$ \\
\hline Postquirúrgico & 41 & $19(46,3)$ & $5(12,2)$ \\
\hline Postraumático & 19 & $3(15,8)$ & 0 \\
\hline Absceso pulmonar & 10 & $7(70,0)$ & $3(30,0)$ \\
\hline Tuberculosis & 10 & $1(10,0)$ & 0 \\
\hline Neoplásico & 6 & $3(50,0)$ & $3(50,0)$ \\
\hline Neumotórax & 2 & 0 & 0 \\
\hline Desconocido & 13 & $6(46,2)$ & $4(30,8)$ \\
\hline
\end{tabular}

Tabla 8. Complicaciones, reoperaciones y requerimientos de UCI post operatoria en pacientes con EP tratados quirúrgicamente

\begin{tabular}{|lrc|}
\hline Complicaciones más frecuentes & & \\
Empiema (colección residual) & 26 & $(7,6 \%)$ \\
Fuga área & 14 & $(4,1 \%)$ \\
Infección herida operatoria & 7 & $(2,0 \%)$ \\
Hemotórax & 5 & $(1,5 \%)$ \\
Insuficiencia respiratoria & 5 & $(1,5 \%)$ \\
Sepsis de origen extrapleural & 21 & $(6,1 \%)$ \\
Reoperaciones & 29 & $(8,5 \%)$ \\
Empiema (colección residual) & 17 & $(4,9 \%)$ \\
Sangrado & 6 & $(1,7 \%)$ \\
Cavidad residual & 2 & $(0,6 \%)$ \\
Otras & 4 & $(1,2 \%)$ \\
Requerimiento de UCI post operatorio & & \\
$\quad$ No & $308(89,8 \%)$ \\
Sí & $35(10,2 \%)$ \\
\hline
\end{tabular}

pneumoniae, Acinetobacter baumannii y otros gérmenes; tipo de cirugía; reoperación y necesidad de UCI. De las variables seleccionadas para la mortalidad presentaron significación estadística en el análisis multivariado: edad mayor de 70 años; comorbilidad neoplásica; fiebre al momento del procedimiento; deterioro de conciencia; etiología del EP; identificación de germen en líquido pleural; cultivo con Staphylococcus aureus, Acinetobacter baumannii y otro germen y necesidad de UCI (Tabla 9).

\section{Discusión}

El EP es una patología evolutiva que tradicionalmente se divide en fases según las características de la ocupación pleural y que se correlacionan con el tiempo de evolución. Estas son: Fase I o exudativa, Fase II o fibrinopurulenta y Fase III de organización o consolidación. Estas fases evolutivas son dinámicas y entre ellas existe un período de transición en que se superponen; para alcanzar la Fase III se requieren entre 5 a 6 semanas de evolución. Esta clasificación del EP permite unificar definiciones, determinar pronósticos y plantear tratamientos $3,4,10,12$.

En la serie presentada esta variable no fue consignada, ya que es un dato que en la revisión retrospectiva resulta impreciso y difícil de obtener, esto principalmente por que la mayoría de los casos corresponden a pacientes derivados desde otros hospitales, donde fueron tratados por un tiempo que generalmente no es precisado. El tiempo de evolución es particularmente difícil de determinar en los casos de empiema paraneumónico ya que los pacientes llevan varias semanas en tratamiento por la neumonía, este hecho asociado a que la mayoría de los casos necesitó una decorticación pleuropulmonar por toracotomía, nos hacen inferir que muchos de los pacientes se encontraban en Fase III y por lo tanto con un tiempo de evolución prolongado.

Estas características de la serie, explicarían el bajo porcentaje de pacientes en quienes se realizó cirugía videotoracoscópica o vídeo-asistida, que es el abordaje de elección en la mayoría de los EP, especialmente en etapa inicial ${ }^{2,3,9}$.

Otra posible consecuencia de esto, es el bajo porcentaje de cultivos microbiológicos positivos que encontramos en líquido pleu- 
Tabla 9. Análisis univariado y multivariado de variables seleccionadas asociados a morbi-mortalidad en pacientes con EP tratados quirúrgicamente

\begin{tabular}{|c|c|c|c|c|}
\hline Variables & $\begin{array}{l}\text { Morbilidad } \\
\text { univariado }\end{array}$ & $\begin{array}{c}\text { Morbilidad } \\
\text { multivariado }\end{array}$ & $\begin{array}{l}\text { Mortalidad } \\
\text { univariado }\end{array}$ & $\begin{array}{l}\text { Mortalidad } \\
\text { multivariado }\end{array}$ \\
\hline Edad $<$ o $>70$ años & NS & NS & $\mathrm{p}<0,05$ & $\mathrm{p}<0,05$ \\
\hline Género $\mathrm{M}$ o $\mathrm{F}$ & NS & NS & NS & NS \\
\hline Ambiente neoplásico & NS & NS & $\mathrm{p}<0,05$ & $\mathrm{p}<0,05$ \\
\hline Diabetes mellitus & $\mathrm{p}<0,05$ & NS & NS & NS \\
\hline Tabaquismo & NS & NS & NS & NS \\
\hline Fiebre & NS & NS & NS & $\mathrm{p}<0,05$ \\
\hline Deterioro hemodinámico & $\mathrm{p}<0,05$ & $\mathrm{p}<0,05$ & $\mathrm{p}<0,05$ & NS \\
\hline Deterioro de conciencia & $\mathrm{p}<0,05$ & $\mathrm{p}<0,05$ & $\mathrm{p}<0,05$ & $\mathrm{p}<0,05$ \\
\hline Taquicardia & NS & NS & NS & NS \\
\hline Etiología & $\mathrm{p}<0,05$ & NS & $\mathrm{p}<0,05$ & $\mathrm{p}<0,05$ \\
\hline Germen aislado & $\mathrm{p}<0,05$ & $\mathrm{p}<0,05$ & NS & NS \\
\hline Germen líquido pleural & $\mathrm{p}<0,05$ & NS & NS & $\mathrm{p}<0,05$ \\
\hline Cultivo polimicrobiano & $\mathrm{p}<0,05$ & NS & NS & NS \\
\hline Staphylococcus aureus & $\mathrm{p}<0,05$ & $\mathrm{p}<0,05$ & $\mathrm{p}<0,05$ & $\mathrm{p}<0,05$ \\
\hline Streptococos spp & NS & NS & NS & NS \\
\hline Pseudomonas aeruginosa & $\mathrm{p}<0,05$ & NS & $\mathrm{p}<0,05$ & NS \\
\hline Klebsiella pneumoniae & $\mathrm{p}<0,05$ & $\mathrm{p}<0,05$ & NS & NS \\
\hline Escherichia coli & NS & NS & NS & NS \\
\hline Acinetobacter baumannii & $\mathrm{p}<0,05$ & $\mathrm{p}<0,05$ & $\mathrm{p}<0,05$ & $\mathrm{p}<0,05$ \\
\hline Otro germen & $\mathrm{p}<0,05$ & $\mathrm{p}<0,05$ & NS & $\mathrm{p}<0,05$ \\
\hline Tipo cirugía & $\mathrm{p}<0,05$ & $\mathrm{p}<0,05$ & NS & NS \\
\hline Reoperación & $\mathrm{p}<0,05$ & $\mathrm{p}<0,05$ & NS & NS \\
\hline UCI & $\mathrm{p}<0,05$ & $\mathrm{p}<0,05$ & $\mathrm{p}<0,05$ & $\mathrm{p}<0,05$ \\
\hline
\end{tabular}

ral $(25,9 \%)$. El tiempo de evolución y tratamiento prolongado en otros hospitales, podrían explicar, al menos en parte, la negativización de los cultivos microbiológicos.

En esta serie quirúrgica de pacientes, el EP fue más frecuente en el género masculino y la edad promedio fue similar a las comunicadas en otras series quirúrgicas de $\mathrm{EP}^{6,8,13-15}$. Las patologías asociadas y en particular las que producen inmunosupresión, se han descrito como factores predisponentes al desarrollo de un EP y se han identificado como factores que se asociarían a morbilidad y mortalidad postquirúrgica ${ }^{4,7,16}$. En nuestra serie se presentan comorbilidades como diabetes mellitus, hipertensión arterial y tabaquismo, pero también se identifican factores que se asocian a gravedad como el deterioro de conciencia y deterioro hemodinámico al momento de la cirugía. Además, el 10,8\% de los pacientes desarrollaron el EP en un "ambiente neoplásico", es decir, en el contexto de una neoplasia maligna ya sea pleuropulmonar o de otro origen.

La etiología más frecuente fue el empiema paraneumónico, seguido de los postquirúrgicos y postraumáticos, similar a lo comunicado $0^{1,3,6,10,13,17}$. El empiema paraneumónico en todas las series es la causa más frecuente de EP, es más frecuente en edades extremas y en la última década han aumentado los casos y la mortalidad en relación principalmente a pacientes con inmunosupresión ${ }^{4,5,11}$.

Los EP postquirúrgicos de cirugía torácica constituyen un grupo de especial interés porque se asocian a mayor morbi-mortalidad y tienen una microbiología considerada más agresiva, principalmente a expensas de Staphylococcus aureus y a gérmenes Gram negativos ${ }^{1,3,10,18,19}$. De los 41 casos de esta serie, destacamos que la mayoría corresponde a 
empiemas postquirúrgicos de otros hospitales que nos fueron derivados para tratar el empiema, sólo 8 casos corresponden a pacientes que fueron operados primariamente de cirugía torácica en nuestro hospital.

Entre el 1,6\% a 4,2\% de los traumatismos torácicos evoluciona con EP, las causas son multifactoriales y son más frecuentes en traumatismos graves $\mathrm{y}$ en el contexto de politraumatismo ${ }^{20-23}$, en nuestra serie el 5,5\% corresponde a EP postraumáticos.

Otras causas de EP como la tuberculosis fueron menos frecuentes, aunque la etiología tuberculosa en algunas series es la segunda después de los EP paraneumónicos. Los EP por tuberculosis se asocian a mayor fibrosis, a peores resultados funcionales postquirúrgicos y corresponden a una entidad distinta al derrame pleural tuberculoso ${ }^{24-26}$.

En EP los gérmenes aislados en el líquido pleural más frecuente son el Staphylococcus aureus y Streptococcus spp, similar a lo encontrado en nuestra serie, sin embargo, lo comunicado es que entre el 50 a $70 \%$ de los cultivos de líquido pleural son positivos ${ }^{1,19,27,28}$, en nuestro caso estos alcanzaron sólo el 25,9\%. Los gérmenes anaerobios están presentes hasta en el $63 \%$ de los cultivos en $\mathrm{EP}^{1,28}$, en nuestro medio la posibilidad de realizar este tipo de cultivo no siempre está disponible y en nuestra serie no se consignaron.

Los objetivos del tratamiento quirúrgico del EP son: tratar la infección drenando la ocupación pleural, lograr adecuada expansión pulmonar y evitar cavidades residuales ${ }^{3,10}$. Estos objetivos se pueden lograr con distintas alternativas quirúrgicas que van desde la pleurotomía hasta la decorticación por toracotomía. En casos excepcionales, especialmente en complicaciones como en fístulas broncopleurales, son necesarias técnicas como la fenestración, toracoplastia y uso de colgajos musculares ${ }^{3,10,29-31}$.

La alternativa seleccionada va a depender de factores como: la fase evolutiva del EP, etiología, comorbilidades o gravedad del paciente y disponibilidad o experiencia del equipo quirúrgico.

La pleurotomía en EP, es un procedimiento quirúrgico que se considera de elección para EP en fases iniciales y también en casos en que existen patologías asociadas graves que contraindiquen la cirugía, en pacientes que tengan expectativas de vida limitada o en que la morbi-mortalidad hagan prohibitivo otro procedimiento más invasivo. Es un procedimiento quirúrgico simple de realizar, requiere implementación básica y es bien tolerado. Se describe como exitoso o suficiente entre el 65 a $75 \%$ de los casos, por lo que entre el 35 a $65 \%$ de los pacientes requieren otro procedimiento quirúrgico. La mortalidad en pacientes tratados exclusivamente con pleurotomía alcanza entre el 10 a $25 \%$, esto a expensas del segundo tipo de pacientes que son los que tienen mayor riesgo o expectativas limitadas de vida $^{3,5,6}$. La mortalidad de los pacientes tratados en nuestra serie exclusivamente con pleurotomía fue de $15,7 \%$.

Los procedimientos mínimamente invasivos como la cirugía videotoracoscópica o video-asistida han adquirido en las dos últimas décadas un importante rol en el tratamiento del EP, hoy en día constituyen la vía de abordaje más utilizada especialmente en EP iniciales. Como procedimiento final o único tiene un éxito entre 68 a 93\%, con necesidad de conversión o de otra cirugía entre el 10 a 40\% de los casos, especialmente en pacientes con empiemas en fase de organización. En pacientes tratados con estas técnicas la mortalidad descrita se encuentra entre el 3,5 a $5 \% \%^{2,3,9,32-34}$. Dadas las características que fueron comentadas, en nuestra serie sólo el 3,2\% (11 pacientes) fueron tratados con esta técnica.

La cirugía tradicional o "abierta" es la decorticación pleuropulmonar por toracotomía. Esta cirugía puede abarcar un amplio espectro de hallazgos y procedimientos quirúrgicos, que van desde un aseo pleural con retiro de material pleural líquido y una coraza pleural incipiente muy fácil de retirar, hasta el retiro de material pleural organizado y una coraza pleural muy adherida y gruesa que puede alcanzar varios centímetros. Esta cirugía es invasiva, requiere implementación y un equipo quirúrgico entrenado, además se puede asociar a otros procedimientos como resección pulmonar. La decorticación pleuropulmonar está especialmente indicada en EP en fases de organización y/o cuando las otras alternativas quirúrgicas no son posibles o suficientes. La morbilidad de la decorticación pleuropulmonar por toracotomía puede alcanzar hasta el 30\% y la mortalidad al $10 \%$. Las complicaciones más frecuentemente descritas son fístulas broncopleurales, infección de herida operatoria, hemorragias post operatorias y

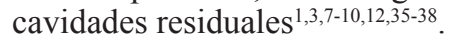

Otras técnicas como la fenestración o la toracoplastía, se dejan para casos extremos en que las otras alternativas quirúrgicas no logran el objetivo de tratar la infección y lograr expansión pulmonar evitando cavidades residuales, de ser posible, deben evitarse ya que se asocian con un deterioro significativo en la calidad de vida de los pacientes ${ }^{3,10,29-31}$.

En EP la terapia con fibrinolíticos continua siendo tema de discusión, su rol e indicaciones aún son motivo de controversia. Parece ser útil si se utiliza precozmente en EP tabicados, particularmente en pacientes con empiema paraneumónico, en casos con alto riesgo quirúrgico y en centros con poca capacidad de resolución quirúrgica ${ }^{1,3-5,39,40}$. El uso de fibrinolíticos no fue una variable consignada en nuestra serie. 
El EP en series quirúrgicas mantiene una morbilidad y mortalidad considerables, la morbilidad puede alcanzar el $30 \%$ y la mortalidad el $20 \%$ en grupos de riesgo $0^{1-9,11,38}$. Nuestra serie de pacientes globalmente tiene una morbilidad de $30,3 \%$ y una mortalidad de $9,0 \%$. La morbi-mortalidad fue mayor en pacientes de algunos grupos como los EP asociados a absceso pulmonar, neoplasia maligna, en pacientes tratados exclusivamente con pleurotomía o en quienes se asoció una resección pulmonar.

En nuestra serie la morbilidad y la mortalidad presentaron variables asociadas en el análisis univariado y multivariado. De estas, la edad mayor de 70 años, comorbilidad neoplásica, fiebre al momento del procedimiento, deterioro de conciencia, deterioro hemodinámico, etiología del EP, identificación de germen en líquido pleural, cultivo con: Staphylococcus aureus, Klebsiella pneumoniae, Acinetobacter baumannii y otro germen, tipo de cirugía y necesidad de reoperación o UCI, también son comunicadas y asociadas a morbi-mortalidad en las series de quirúrgicas de $\mathrm{EP}^{1,3,7,16,35,38}$.

Por todo lo anteriormente comentado (derivación desde otros hospitales, tiempo de evolución, fases de empiema, etiologías, tipos de cirugías, morbi-mortalidad, etc.) y teniendo presente que nuestro hospital es un centro de referencia de alta complejidad, creemos que esta serie de EP adquiere características especiales, tratándose de pacientes seleccionados, que corresponden a un espectro de EP que podríamos catalogar como avanzados o complejos.

A modo de conclusiones podemos señalar que en EP tratados quirúrgicamente: el empiema paraneumónico es el tipo más frecuente, son más frecuentes en el género masculino, en un bajo porcentaje se identifican gérmenes en líquido pleural, la decorticación por toracotomía es el procedimiento quirúrgico más frecuente y que el tratamiento quirúrgico no está exento de morbilidad ni mortalidad. En nuestra serie se identifican variables asociadas a morbimortalidad.

\section{Agradecimientos}

A nuestras secretarias Sra. Laura Cortés A. y Sra. Lidia León F., por su constante y desinteresado apoyo en la preparación de esta comunicación.

\section{Referencias}

1. Brims FJ, Lansley SM, Waterer GW, Lee YC. Empyema thoracis: new insights into an old disease. Eur Respir Rev. 2010;19:220-8.

2. Ahmed AE, Yacoub TE. Empyema thoracis. Clin Med Insights Circ Respir Pulm Med. 2010;4:1-8.
3. Molnar TF. Current surgical treatment of thoracic empyema in adults. Eur J Cardiothorac Surg. 2007;32:42230.

4. Koegelenberg CFN, Diacon AH, Bolliger CT. Parapneumonic pleural effusion and empyema. Respiration 2008;75:241-50.

5. Light RW. Parapneumonic effusions and empyema. Proc Am Thorac Soc. 2006;3:75-80.

6. Salguero J, Cardemil G, Molina J, Lembach H, Fernández R. Empiema Pleural: Etiología, tratamiento y complicaciones. Rev Chil Cir. 2009;61:223-8.

7. Hsieh MJ, Liu YH, Chao YK, Lu MS, Liu HP, Wu YC, et al. Risk factors in surgical management of thoracic empyema in elderly patients. ANZ J Surg. 2008;78:4458.

8. Bar I, Stav D, Fink G, Peer A, Lazarovitch T, Papiashvilli $\mathrm{M}$. Thoracic empyema in high-risk patients: conservative management or surgery? Asian Cardiovasc Thorac Ann. 2010;18:337-43.

9. Chambers A, Routledge T, Dunning J, Scarci M. Is video-assisted thoracoscopic surgical decortication superior to open surgery in the management of adults with primary empyema? Interact Cardiovasc Thorac Surg. 2010;11:171-7.

10. Christie NA. Management of pleural space: effusions and empyema. Surg Clin North Am. 2010;90:919-34.

11. Bender JM, Ampofo K, Sheng X, Pavia AT, CannonAlbright L, Byington CL. Parapneumonic empyema deaths during past century, Utah. Emerg Infect Dis .2009; $15: 44-8$.

12. Shiraishi Y. Surgical treatment of chronic empyema. Gen Thorac Cardiovasc Surg. 2010;58:311-6.

13. Roberts JR. Minimally invasive surgery in the treatment of empyema: intraoperative decision making. Ann Thorac Surg. 2003;76:225-30.

14. Yamaguchi M, Takeo S, Suemitsu R, Matsuzawa H, Okazaki H. Video-assisted thoracic surgery for fibropurulent thoracic empyema: a bridge to open thoracic surgery. Ann Thorac Cardiovasc Surg. 2009;15:368-72.

15. Tacconi F, Pompeo E, Fabbi E, Mineo TC. Awake video-assisted pleural decortication for empyema thoracis. Eur J Cardiothorac Surg. 2010;37:594-601.

16. Nielsen J, Meyer CN, Rosenlund S. Outcome and clinical characteristics in pleural empyema: A retrospective study. Scand J Infect Dis. 2011;43:430-5.

17. Manunga J, Olak J. Is thoracoscopic decortication sufficient for the treatment of empyema? Am Surg. 2010;76:1050-4.

18. Chen W, Lin YC, Liang SJ, Tu CY, Chen HJ, Hang LW, et al. Hospital-acquired thoracic empyema in adults: a 5-year study. South Med J. 2009;102:909-14.

19. Meyer CN, Rosenlund S, Nielsen J, Friis-Möller A. Bacteriological aetiology and antimicrobial treatment of pleural empyema. Scand J Infect Dis. 2011;43:165-9.

20. Aguilar MM, Battistella FD, Owings JT, Su T. Posttraumatic empyema. Risk factor analysis. Arch Surg. 
1997;132:647-50.

21. Mandal AK, Thadepalli H, Mandal AK, Chettipalli U. Posttraumatic empyema thoracis: a 24-year experience at a major trauma center. J Trauma 1997;43:764-71.

22. Burton PR, Lee M, Bailey M, Pick AW. What causes post-traumatic empyema? Emerg Med Australas. 2009;21:153-9.

23. Carr JA, Fales C, Shaikh IA, Foulds K. Computed tomographic modeling before and after treatment for posttraumatic empyema: early decortication is superior to catheter drainage. Ann Thorac Surg. 2011;91:1723-8.

24. Sahn SA, Iseman MD. Tuberculous empyema. Semin Respir Infect. 1999;14:82-7.

25. Malhotra P, Aggarwal AN, Agarwal R, Ray P, Gupta D, Jindal SK. Clinical characteristics and outcomes of empyema thoracis in 117 patients: a comparative analysis of tuberculous vs. non-tuberculous aetiologies. Respir Med. 2007;101:423-30.

26. Kundu S, Mitra S, Mukherjee S, Das S. Adult thoracic empyema: A comparative analysis of tuberculous and nontuberculous etiology in 75 patients. Lung India 2010;27:196-201.

27. Clark J. Microbiology and management of pleural empyema. Adv Exp Med Biol. 2009;634:61-8.

28. Chen KY, Hsueh PR, Liaw YS, Yang PC, Luh KT. A 10-year experience with bacteriology of acute thoracic empyema: emphasis on Klebsiella pneumoniae in patients with diabetes mellitus. Chest 2000;117:1685-9.

29. Regnard JF, Alifano M, Puyo P, Fares E, Magdeleinat P, Levasseur P. Open window thoracostomy followed by intrathoracic flap transposition in the treatment of empyema complicating pulmonary resection. J Thorac Cardiovasc Surg. 2000;120:270-5.

30. Jiang L, Jiang GN, He WX, Fan J, Zhou YM, Gao W, et al. Free rectus abdominis musculocutaneous flap for chronic postoperative empyema. Ann Thorac Surg. 2008;85:2147-9.

31. Massera F, Robustellini M, Della Pona C, Rossi G, Rizzi A, Rocco G. Open window thoracostomy for pleural empyema complicating partial lung resection. Ann Thorac Surg. 2009;87:869-73.

32. Colice GL, Curtis A, Deslauriers J, Heffner J, Light R, Littenberg B, et al. Medical and surgical treatment of parapneumonic effusions : an evidence-based guideline. Chest 2000;118:1158-71.

33. Tong BC, Hanna J, Toloza EM, Onaitis MW, D'Amico TA, Harpole DH, et al. Outcomes of videoassisted thoracoscopic decortication. Ann Thorac Surg. 2010;89:220-5.

34. Zahid I, Nagendran M, Routledge T, Scarci M. Comparison of video-assisted thoracoscopic surgery and open surgery in the management of primary empyema. Curr Opin Pulm Med. 2011;17:255-9.

35. Melloni G, Carretta A, Ciriaco P, Negri G, Voci C, Augello G, et al. Decortication for chronic parapneumonic empyema: results of a prospective study. World J Surg. 2004;28:488-93.

36. Pilav I, Guska S, Musanovic S, Kadric K. Surgical treatment of pleural empyema according to disease stage. Med Arh. 2009;63:291-4.

37. Lee SF, Lawrence D, Booth H, Morris-Jones S, Macrae B, Zumla A. Thoracic empyema: current opinions in medical and surgical management. Curr Opin Pulm Med. 2010;16:194-200.

38. Rodríguez A, Lisboa T, Blot S, Martin-Loeches I, Solé-Violan J, De Mendoza D, et al. Mortality in ICU patients with bacterial community-acquired pneumonia: when antibiotics are not enough. Intensive Care Med. 2009;35:430-8.

39. Misthos P, Sepsas E, Konstantinou M, Athanassiadi K, Skottis I, Lioulias A. Early use of intrapleural fibrinolytics in the Management of postpneumonic empyema. A prospective study. Eur J Cardiothoracic Surg. 2005;28:599-603.

40. Maskell NA, Davies CW, Nunn AJ, Hedley EL, Gleeson FV, Miller R, et al. U.K. Controlled trial of intrapleural streptopkinase for pleural infection. N Engl J Med. 2005;352:865-74. 